\title{
LIBRE MERCADO Y REGULACIÓN: LA EXPERIENCIA EN EL SECTOR ELÉCTRICO ESPAÑOL
}

\author{
MARKET AND REGULATION: THE EXPERIENCE IN THE SPANISH \\ ELECTRICITY SECTOR
}

\author{
Alberto Olivares Gallardo*
}

\begin{abstract}
RESUMEN: Se describe a la regulación como instrumento de intervención administrativa en un panorama de liberalización de los servicios económicos esenciales, en especial del sector eléctrico. Desde esta perspectiva, se examina la transformación que ha sufrido la relación del Estado con la sociedad, producto del nuevo paradigma económico, que promueve un entorno liberalizado para el suministro de la electricidad, regulado por organismos independientes que intervienen el mercado energético, sin participar en él. Finalmente, se analiza la complementación que existe entre el organismo regulador independiente de energía y el sistema de defensa de la libre competencia, con la finalidad de lograr el adecuado funcionamiento de los mercados energéticos. El estudio se hace desde la evolución del sector eléctrico español.
\end{abstract}

Palabras clave: Libre mercado, regulación, sector eléctrico.

\begin{abstract}
This paper refers to the regulation as an instrument of administrative intervention in an overview of liberalization of essential utilities, especially in the electricity industry. From this perspective, we examined the transformation undergone by the state's relationship with society, due to the new economic paradigm, which promotes a liberalized economy for the electricity supply, regulated by independent agencies that intervene in the energy market, without participating in it. Finally, we analyse the relationship between the independent energy regulator and the antitrust system, with the purpose of achieving the suitable functioning of energy markets. The study was done from the evolution of the Spanish electricity sector.
\end{abstract}

Key words: Open market, regulation, electricity industry.

\section{A MODO DE INTRODUCCIÓN}

La energía, como capacidad para realizar un trabajo ${ }^{1}$, se encuentra en la naturaleza bajo diversas formas. Estas múltiples manifestaciones de la energía, denominadas fuentes energéticas, pueden ser clasificadas en primarias y secundarias, según si se obtienen directamente de la naturaleza o si requieren de un proceso de transformación. Así, son fuentes primarias de energía: el petróleo, el carbón mineral, el gas natural, el viento, el sol, el agua, la energía nuclear, entre otros; por otra parte, son ejemplos de energías secundarias los derivados del petróleo o la electricidad.

\footnotetext{
* Docente de Derecho Público e Investigador responsable del Grupo de Investigaciones Jurídicas, Universidad Católica de Temuco. Correo electrónico: alberto.olivares@uct.cl.

1 Definición de energía de la Real Academia Española (2011).
} 
Los recursos energéticos primarios pueden ser renovables o no renovables. En este sentido, Medlock explica que: "A resource is considered depletable when the sum over time of all possible production is finite, or the stock of the resources is not replaceable in a reasonable timeframe. Crude oil, natural gas, and coal-fossil fuels- are examples of depletable resources. Non-depletable energy resources include geothermal, wind, and solar" ${ }^{2}$.

Históricamente, encontramos que la electricidad ha sido generada desde diversas fuentes energéticas. Sucintamente, puede decirse que en un comienzo su producción se restringía casi exclusivamente al carbón y el agua. A partir de la segunda mitad del siglo XX el petróleo y la energía nuclear pasaron a ser opciones del combinado energético en los diversos países. Finalmente, durante el último cuarto del siglo XX, el gas natural y los recursos energéticos autóctonos (renovables) han conformado la estructura actual de la generación de electricidad.

Desde una perspectiva física, la electricidad es un vector energético que se presenta como esencial en nuestra época ${ }^{3}$. Es requerido para la mayor parte de las acciones que realizamos en nuestra vida diaria y está presente en casi todas las actividades productivas y de servicios.

Por otra parte, el suministro eléctrico como actividad se realiza en fases sucesivas; situación que permite su funcionamiento como un sistema. Esta singularidad de la electricidad ha estimulado naturalmente la integración vertical de las diversas empresas que operan en la generación, el transporte, la distribución y la comercialización, que constituyen las cuatro fases del suministro eléctrico ${ }^{4}$.

Realizadas estas pequeñas explicaciones, de carácter introductorio, debe señalarse que el presente trabajo está dedicado a la actividad del sector eléctrico. Más en particular, se estudiará la regulación como técnica de intervención administrativa a que es sometida la industria. La principal motivación para la regulación la encontramos en el conjunto de características técnicas y económicas propias de la actividad eléctrica, entre las que destacan: la imposibilidad de almacenamiento, la constitución histórica de su industria como monopolio natural en torno a empresas integradas verticalmente $y$, que requiere de un complejo sistema de redes para su suministro.

Estas características han condicionado la relación de la administración con el sector, que ha ido modificándose a lo largo del tiempo, siendo posible identificar, de manera muy general, tres grandes períodos. En primer lugar, a finales del siglo XIX, época de su génesis, la industria eléctrica se encontraba en manos privadas, y era ejercida en un ámbito de libertad empresarial. La intervención del Estado se limitó, en esta primera época, a la

\footnotetext{
2 Medlock (2009) p. 51.

3 Rivero (2009) p. 79.

${ }^{4}$ La industria eléctrica de cuatro fases (generación, transporte, distribución y comercialización o suministro) pertenece a una segunda etapa de liberalización del sector. Esta organización es propia del sector energético de los Estados miembros de la Unión Europea. En Chile, en cambio, el sistema eléctrico se organiza como una industria de tres fases -generación, transporte y distribución-, propia de la primera etapa del proceso de liberalización iniciado en los años 80 con el DFL No1/1982, que aprueba una nueva "Ley General de Servicios Eléctricos" (LGSE). En este modelo, el suministro al consumidor final es realizado por el distribuidor. Sobre el marco regulatorio del sistema eléctrico nacional, véase VERGARA (2004).
} 
tributación de la incipiente industria, a la servidumbre de paso de la red de transporte, a cuestiones de seguridad de las instalaciones y a una embrionaria protección de los usuarios ante abusos de las empresas 5 . Sobre este tema, apunta Nebreda Pérez: "Desde los albores del suministro eléctrico, la Administración tuvo claro que la nueva actividad industrial era un importante centro recaudatorio, que requería de alguna protección en favor de sus consumidores, y que su implantación, socialmente beneficiosa, tenía evidentes dificultades a superar" ${ }^{\prime}$. Se comenzó a realizar, por tanto, una intervención administrativa casuística, sectorial y desorganizada.

Una segunda etapa reconocible de la relación entre la Administración y el sector eléctrico imperó desde la década de los 40 hasta finales del siglo XX, expresado en la institución del servicio público. La reconstrucción de Europa en el período de entreguerras, y con mayor fuerza en la posguerra, trae consigo la planificación y concreción de un proceso de nacionalización de los servicios económicos de interés general, entre ellos el que especialmente nos preocupa, la industria eléctrica ${ }^{7}$. Sobreviene al periodo inicial, en que la industria estaba en manos privadas con relativamente poca regulación del Estado, una etapa de fortalecimiento y desarrollo del Estado prestador de servicios, que asume directamente y en monopolio, la gestión de los servicios públicos económicos ${ }^{8}$.

Por tanto, para el caso del sector eléctrico, fue este fenómeno -la nacionalización, como instrumento de reconstrucción de la Europa de posguerra- un elemento decisivo en la transformación de la actividad de policía o de limitación del Estado -que se expresaba en regulación y control de la actividad para su buen funcionamiento- hacia una prestación y gestión de la actividad eléctrica, a través de la técnica del servicio público. Es en este período en que se configuran con sus estructuras actuales las empresas eléctricas de los diversos países, así como el objetivo de los Estados de organizar la electricidad como un sistema?.

Finalmente, el ocaso de la institución del servicio público y la irrupción de la liberalización del sector energético, da inicio a una tercera etapa en la intervención de la Administración en la industria eléctrica, caracterizada por el retraimiento del Estado en la prestación de servicios, y la asunción de un nuevo rol, como regulador y garantizador de los derechos de las personas ${ }^{10}$. La reformulación de la clásica institución del servicio público y

\footnotetext{
5 En concreto, para el caso español, un conjunto de normas nos muestran estos objetivos de regulación inicial: el RD 28-V-1896; la Ley de 22-VI-1898; la Ley de 23-III- 1900, desarrollada por el RD de 15-VI-1901; la Ley de 23-III- 1900; el RD de 25-I-1901; el RD de 22-XI-1912; entre otros. Para profundizar sobre la legislación eléctrica de la época, Véase Gimeno (1994); Trillo-Figueroa y López-Jurado (1996).

${ }^{6}$ Nebreda Pérez (1999) p. 51.

7 Si bien la nacionalización del sector eléctrico fue un proceso más o menos generalizado, debe tenerse en cuenta la advertencia de MuÑoz MACHADO, en el sentido de que si bien países como Francia e Inglaterra llevaron a cabo sus procesos de nacionalización, convencidos de la necesidad de configurar una red única y organizar la electricidad como sistema, España mantuvo el régimen privado de la producción de la electricidad, dando el carácter de servicio público a su suministro (en el RD de 12 de abril de 1924), en MuÑoz (1998) p. 31.

8 CHICK (2007) p. 84.

9 Ariño y López (1998) pp. 46 y ss.

10 Bacigalupo (2009-A) p. 93.
} 
su adaptación a los nuevos tiempos, a través de la idea de servicios de interés económico ${ }^{11}$, trajo consigo la reestructuración no solo de toda la actividad administrativa que incidía sobre los sectores económicos, sino que también -de manera específica- significó la reformulación de la noción de regulación, que abandona su concepción clásica (influenciada por el Derecho europeo continental), centrado en la capacidad normativa del Estado, para moldearse a las nuevas ideas liberalizadoras que comienzan a aparecer a finales del siglo pasado.

Es en este proceso actual de redefinición del rol del Estado en su relación con la sociedad, provocado por un verdadero cambio de paradigma en la economía, que se sitúa el presente trabajo. La reconfiguración de la regulación, como técnica de intervención de la Administración, en un proceso de liberalización de los sectores económicos esenciales, entre ellos el eléctrico, ha significado una verdadera aproximación de la regulación a la concepción anglosajona de regulation, menos centrada en la actividad normativa y con énfasis en una intervención integral de control. "Del paradigma de la confianza en el acierto de las autoridades políticas y administrativas para limitar la propiedad y la libertad de empresa al servicio de los intereses públicos; se va a evolucionar a una confianza progresiva en la libre competencia, como un medio más eficaz que la intervención pública para obtener una mayor eficiencia económica que beneficia a toda la comunidad, a través de la funcionalidad social y económica de los derechos de propiedad y de la libertad de empresa"12.

Por tanto, esta nueva acepción de la noción de regulación, propia de las economías liberalizadas, y su influencia en la industria energética será aquí estudiada, desde la perspectiva que entrega el Derecho. En este sentido, se pretende exponer que la regulación, como técnica de intervención administrativa propia del Estado actual en una economía liberalizada, ha configurado un nuevo estatuto jurídico para la industria eléctrica, con énfasis en la libertad de empresa, pero con tareas pendientes en materia de protección al consumidor y de libre competencia, que se expresa por ejemplo en la transformación de las grandes estructuras nacionales, consolidadas a lo largo del siglo XX -campeones nacionales los llama Ariño ${ }^{13}$-, en organizaciones regionales aún más poderosas, que actúan en el mercado eléctrico de la Unión Europea, los campeones europeos.

\section{CARACTERÍSTICAS DE LA ELECTRICIDAD Y DE LA ACTIVIDAD ELÉCTRICA}

Lo primero que debe tenerse presente al momento de iniciar cualquier análisis jurídico de la energía eléctrica, es que tiene una naturaleza particular y presenta dificultades

\footnotetext{
11 Los servicios públicos, como prestaciones generales a la colectividad, en forma generalmente de infraestructuras, que proporcionan una calidad de vida mínima a todos los ciudadanos, admiten diversas clasificaciones, siendo pertinente recordar -para efectos de este estudio- aquella distinción material entre servicios públicos sociales y económicos, correspondiendo los primeros a aquella actividad estatal de beneficencia o asistencial (asistencia sanitaria, educación primaria, etc.), y los segundos al desarrollo de ciertas actividades de contenido sustancialmente económico, y que son considerados como un valor añadido al bienestar general (energía, telecomunicaciones, etc.). Véase ARIÑo (2001) pp. 125 y ss.; ARIÑo (1993) pp. 68 y ss.; Muñoz (1998) pp. 862 y ss.; Cassagne (1996) pp. 98 y ss.

12 De la Cruz (2002) p. 6.

13 ARIÑo (2010) p. 151.
} 
intrínsecas para ser comprendida desde la experiencia común de las personas. No es equiparable, en sus propiedades físicas, a ningún otro bien que pueda ser suministrado, tampoco a ningún otro servicio que pueda ser prestado $^{14}$. Desde esta perspectiva, la electricidad como vector energético presenta un conjunto de particularidades técnicas, y su industria un cúmulo de características económicas, que hacen del suministro eléctrico una actividad altamente técnica y compleja.

Estas singularidades físicas y económicas condicionan no solo las diversas actividades que se desarrollan en este sector, sino que también la estructura de sus mercados e influyen sobremanera a la hora de confeccionar su régimen jurídico ${ }^{15}$. Por ello, a pesar de que no constituye el objeto de este trabajo, serán mencionados aquí, para proceder en los siguientes títulos a profundizar sobre la regulación como característica del modelo liberal que se impone en la prestación de los servicios económicos de interés general, entre ellos el suministro eléctrico. Se sostiene en este trabajo que la intensidad de la regulación que recae sobre el sector eléctrico, se debe, precisamente, a las características técnicas y económicas de la electricidad y su sector, que hacen difícil el desarrollo natural de su mercado.

La electricidad es un vector energético producido desde fuentes de generación muy diversas, y a partir de tecnologías igualmente disímiles. Ninguna tecnología es idónea para producir -en condiciones de eficiencia- toda la energía que es demandada por la socie$\mathrm{dad}^{16}$. Como consecuencia, existe una exigencia intrínseca para los Estados y para la industria que proporciona en definitiva este bien, de no prescindir - a priori- de ninguna de las fuentes de generación. En principio, mientras mayor combinación energética posea un Estado, menor dependencia energética tiene de otros países. Asimismo, las altas cuotas de consumo de electricidad que exige el modelo de sociedad actual, hacen imposible que los países puedan cimentar sus políticas energéticas en la explotación de una sola fuente primaria. Las legislaciones de los Estados miembros y también de otros países como Chile, Estados Unidos e incluso China, han tenido en consideración esta premisa y han elaborado estatutos jurídicos que promueven el desarrollo de las energías autóctonas para sumarse a las energías tradicionales en la configuración de sus combinaciones energéticas (energy mixes).

Por la naturaleza de este trabajo, no se pretende agotar el estudio de las características técnicas y económicas, con la enumeración que realizamos a continuación. Más bien se trata de identificar las principales de estas particularidades, que tienen interés para el estudio jurídico sobre la electricidad que aquí se propone, en cuanto condicionan la actividad eléctrica y su modelo de explotación; y este a su vez, influirá decisivamente en el modelo de regulación ${ }^{17}$.

Entre las características técnicas de la electricidad, señalaremos aquellas tres que tienen especial relevancia para nosotros:

a) la imposibilidad de almacenar electricidad en cantidades importantes, debido a la naturaleza física de este recurso energético;

\footnotetext{
14 Fabra (2004) p. 57.

15 De la Cruz (1999) pp. 17-18.

16 Fabra (2004) p. 75.

17 Ariño y López (1998) p. 29.
} 
b) la exigencia de un sistema de redes para su transmisión desde las centrales de generación hasta el consumidor final, y;

c) la necesidad de diversificación de las fuentes de generación de electricidad.

No son las únicas particularidades de tipo técnico, pero las destacamos por cuanto influyen en decisiones esenciales para el modelo energético que desea implementar un Estado.

En cuanto a las características económicas, especial interés desde el punto de vista jurídico tienen:

a) La imprevisibilidad de la demanda eléctrica;

b) La rigidez de la demanda, y;

c) La presencia de importantes barreras de entrada al mercado eléctrico.

Finalmente, se señalan dos características de la electricidad y el sector que desarrolla la actividad eléctrica, que identificaremos como propiamente jurídicas, por nacer de la intervención que ha realizado la Administración sobre la industria, a fin de regularla y dotarse de potestades públicas frente a esta. Estas son:

a) El suministro eléctrico está gravado con una obligación de servicio público;

b) Intensa regulación del sector, expresado en un amplio y técnico ordenamiento jurídico, que reglamenta de manera exhaustiva las diversas actividades eléctricas.

Es en el contexto de esta última característica que se desarrolla el presente estudio, dedicado a la regulación como actividad predominante del Estado en un entorno liberalizado, y en especial a la influencia de esta técnica de intervención administrativa en la configuración actual de la industria eléctrica, que comenzará a analizarse a continuación.

\section{LA REGULACIÓN EN UN ENTORNO LIBERALIZADO}

Comenzaremos distinguiendo dos posibles acepciones del término regulación. En primer lugar, la regulación como construcción del Derecho europeo continental está referida a toda actividad general de elaboración de reglas jurídicas destinadas, en nuestro caso, a proporcionar un estatuto jurídico al sistema eléctrico. Como tal, la regulación ha sido utilizada como instrumento de intervención del sector energético, desde muy temprano por el Estado, para establecer impuestos a una actividad que se observaba como muy lucrativa, proteger a los consumidores, crear normas de seguridad, y regular aspectos relativos a la implantación de las redes de transporte en el territorio ${ }^{18}$. Pronto, la irrupción del servicio público, condicionó la regulación (como creación de reglas jurídicas) hacia el establecimiento de un estatuto que consolidara la posición de preeminencia del Estado que se adjudicaba un conjunto de prerrogativas sobre la industria eléctrica, dado el carácter esencial que su

18 Nebreda (1999) p. 57. 
suministro rápidamente adquirió ${ }^{19}$. Este sentido tradicional de la regulación pervivió en la Europa continental desde el nacimiento de la actividad energética hasta finales del siglo XIX y durante el siglo XX.

Una segunda acepción de regulación comienza a ser utilizada a partir los años 80 , y desde entonces se arraiga en el acervo jurídico de los servicios económicos esenciales en un entorno de liberalización. En este alcance más innovador, proveniente del Derecho anglosajón, la actividad reguladora más que a la elaboración de normas está orientada a la labor de vigilancia, inspección y control, que ahora no recae directamente en la Administración General del Estado, sino en organismos técnicos independientes, que supervigilan sectores económicos concretos en un entorno liberalizado y de mercado.

La regulation se impone como instrumento de intervención en aquellos Estados que promueven la liberalización de las public utilities. La vigilancia y control del correcto funcionamiento de mercados competitivos pasa a manos de las regulatory agencies, organismos de la Administración, de carácter independiente y eminentemente técnico. Hablamos ahora de la regulación como nuevo instrumento de intervención pública o elemento transformador del Derecho Administrativo ${ }^{20}$.

Este modelo anglosajón de intervención administrativa es rápidamente replicado por la Comunidad Europea, y se expresa en el retraimiento del Estado en la gestión de actividades económicas de interés general, en favor de las empresas privadas, y en el fomento de mercados competitivos para estas actividades ${ }^{21}$. En materia energética, el primer paso que da la Comunidad hacia la liberalización de los mercados eléctricos nacionales de los Estados miembros (con vistas a la configuración de un mercado interior de la electricidad), se plasma en la relevante Directiva 96/92/CE, sobre normas comunes para el mercado interior de la electricidad, que desvela, sin ambigüedades, el modelo energético que la Comunidad Europea desea para los Estados miembros y para sí misma.

19 En España, el Real Decreto de 12 de abril de 1924, en el artículo 1o señalaba: "Se declaran servicios públicos los suministros de energía eléctrica, agua y gas a los abonados de las empresas de distribución, correspondiendo al Ministerio de Trabajo, Comercio e Industria la reglamentación de tales servicios para garantía de la seguridad e interés públicos, sin perjuicio de las demás intervenciones que puedan corresponder a otros Departamentos, a las Provincias y al Municipio sobre las concesiones y contratos administrativos". Véase SALAS (1977) pp. 3 y ss.; PAREjo (2010) pp. 566 y ss.

20 Idea sugerida por el profesor de Derecho Administrativo de la Universidad Autónoma de Barcelona Dr. Carles Padrós, en entrevistas realizadas durante el segundo semestre de 2011.

21 En realidad estamos hablando de dos procesos que están interrelacionados: la privatización y la liberalización. Privatización, en cuanto las actividades económicas esenciales pasan del Estado a manos privadas, lo esencial es la transferencia de la propiedad de la industria, desde el Estado hacia la iniciativa privada, En cambio, la liberalización, implica una apertura al mercado de estas actividades, o si se prefiere, una disminución en el control de la industria por la Administración. Debemos tener presente que puede haber privatización sin liberalización, cuando un monopolio estatal pasa a ser monopolio privado (como sucede a nuestro juicio en Chile en el sector eléctrico, pero esto deberá ser objeto de otro estudio), y también puede haber liberalización sin privatización, como actualmente sucede con el sector eléctrico francés, en que EDF, sin dejar de ser un monopolio estatal (aunque ahora posee participación privada en su capital) ha debido (invitado por la UE) liberalizar el mercado francés, permitiendo la competencia en libre mercado de suministradores de otros Estados miembros, de acuerdo con la idea del mercado interior de electricidad. Sobre este punto véase Thomé (2001) pp. 36 y ss. 
Esta Directiva, al igual que lo hecho por la Electricity Act de 1989, de Inglaterra y Gales (norma de referencia para la liberalización de los mercados eléctricos ${ }^{22}$ ), reconoce que entre los principales problemas a resolver, para la liberalización progresiva de los sectores energéticos, se encuentran: la existencia de características monopólicas en las actividades de transmisión y distribución y, la integración vertical de las empresas eléctricas que realizaban la generación, el transporte y la distribución.

La Unión Europea y los Estados miembros comienzan, en consecuencia, a centrar la regulación económica del sector eléctrico en la separación de actividades. En este sentido, la Directiva 96/92/CE y adoptaron dos técnicas esenciales para dar inicio a una real y progresiva, aunque contenida, liberalización de los mercados energéticos de los Estados miembros: i) el acceso de terceros a la red (Third Party Access), y; ii) la separación de las empresas eléctricas integradas, para sus actividades de generación, transmisión y distribución ${ }^{23}$.

Con este cambio radical de la relación del Estado con la economía, que operó en las últimas décadas del siglo XX, se inició un proceso de privatización de muchas empresas públicas y la mayor parte de los servicios públicos se liberalizaron, abriéndose a la libre competencia; entre ellos, el suministro eléctrico. El Estado prestador dio paso al Estado garante (Gewahrlistungstaad), que en esencia vigila "[...] el comportamiento de los mercados para que los operadores respeten la competencia y las regulaciones públicas establecidas en normas, programas o directivas de actuación formuladas por los poderes públicos competentes para asegurar la salvaguardia de los intereses generales y el cumplimiento, en su caso, de las misiones de servicio público que se les confíen" ${ }^{24}$.

La liberalización trajo consigo una nueva forma de actuación de la Administración, más cercana a los administrados y más pragmática. El Estado sufre una profunda revisión de sus finalidades, pero especialmente de los medios y técnicas que emplea para cumplirlas. Se habla de un nuevo equilibrio entre Estado y sociedad, donde el primero reduce su campo de influencia en la prestación de los bienes y servicios, pero incrementa, como contrapartida, su poder de regulación y de vigilancia sobre los mercados ${ }^{25}$.

En este contexto, Ariño habla de "regulación económica", para identificar este estadio del concepto, en que se pone énfasis en la intervención de la Administración en los diversos sectores económicos (en muchos casos servicios públicos), para establecer requisitos univer-

\footnotetext{
22 No podemos dejar de apuntar que en estricto rigor, las primeras notas de la experiencia liberalizadora en la industria energética fueron aportadas por Chile, pero entre los autores es lugar común señalar el nacimiento de la liberalización del sector eléctrico en Inglaterra u Estados Unidos, quizás por la poca importancia de este primer mercado (desde el punto de vista del tamaño) y la absoluta relevancia de las instituciones surgidas de la legislación anglosajona, como el Third Party Access o el Unbundling (separación de actividades). Cfr. ARIÑo (2006) pp. 261 y ss.; en el mismo sentido, Aranzadi señala: "En todo caso, el desarrollo de los nuevos marcos institucionales liberalizados del sector eléctrico, iniciado en Chile en 1978 y extendido sobre todo en los años 90 (Inglaterra y Gales 1990; Noruega 1991, para luego propagarse a Suecia, Australia, Nueva Zelanda y algunos Estados de Estados Unidos)", AranZadi (2006) p. 100.

${ }^{23}$ Estas dos técnicas se mantuvieron en las Directivas 2003/54/CE (que derogó la Directiva 96/92/CE) y 2009/72/CE (que derogó la Directiva 2003/54/CE). Entonces, la regulación energética, se construyó en torno a estos dos principios básicos, para introducir competencia en los mercados energéticos.

24 MuÑoz (2011) pp. 498-499.

25 Mir (2004) p. 35.
} 
sales de entrada y salida de la actividad, fijar condiciones económicas comunes a los actores y someter al sector, en la medida de lo posible, a finalidades públicas superiores ${ }^{26}$.

Asume la regulación una nueva configuración como institución cardinal del modelo de mercado sobre el que se forja la construcción teórica del Estado actual. El repliegue de la Administración, en cuanto a su función de gestora de servicios públicos, da una nueva entidad a la noción, impregnándola de postulados liberales que impulsan la participación activa y relevante de la iniciativa privada en la prestación de servicios económicos generales (energía, agua, telecomunicaciones, transportes, etc.) en un régimen de competencia.

En el Estado garante, la función reguladora aparece como la de mayor importancia, en un modelo que pregona la libre competencia y consolida al mercado como mecanismo de asignación de recursos. Expresa Parejo que, en este sentido, regulación significa, "[...] control estatal de sectores de la economía en los que, tras la pertinente operación de liberalización-privatización, debe funcionar el mercado"27.

En un proceso en que la Administración se reinventa, para estar a la altura de los tiempos y ofrecer un servicio eficiente, de calidad, a precios asequibles y cercano a los ciudadanos, como asimismo para acoger los postulados de la economía liberal imperantes, la regulación aparece como el instrumento más eficaz del Estado para intervenir en el mercado cuando este no pueda actuar de manera eficiente. En palabras de Muñoz: "El Estado pierde posiciones como gestor de empresas y las gana como regulador del mercado. Esta función obtiene ahora el mayor protagonismo. Consiste en vigilar el funcionamiento del mercado, corregir sus 'desfallecimientos', establecer reglas cuando son necesarias para el buen funcionamiento de algunos sectores y, desde luego, asegurar la competencia libre en todos ellos"28.

En este nuevo estadio de la institución, aparece un sello distintivo de la actividad reguladora del Estado, y es que debe ser ejercida de manera separada a la función de gestión ${ }^{29}$. La Administración puede concurrir al mercado, pero en condiciones de igualdad con los particulares y en un régimen de libre competencia, que constituye la esencia del nuevo orden económico.

Siguiendo la idea anterior, Tornos señala que "el proceso de liberalización de la economía tuvo como uno de sus primeros objetivos conseguir establecer una clara delimitación entre las funciones de regulación y actuación. La apertura de sectores económicos a la libre concurrencia (desde los medios de comunicación, el correo, la electricidad, gas, agua o petróleo hasta los servicios funerarios) no comportaría una verdadera concurrencia si una de las partes que podía seguir actuando en el sector liberalizado mantenía el poder de establecer las reglas del juego" ${ }^{30}$.

La separación de las figuras de regulador y regulado - uno de los pilares del nuevo modelo de Estado gestor- se ha solventado a través del desarrollo de autoridades regula-

\footnotetext{
26 Ariño (2001) p. 561.

27 Parejo (2010-A) p. 62.

28 MuÑoz (2011) p. 1010.

29 Señala MuÑoz MACHADO que: "en todos los sectores liberalizados se está implantando una regla general de organización que exige que la función de regulación, ejercida en los servicios públicos tradicionales por la propia Administración titular del servicio, se separe de la función de gestión del servicio, empresa o actividad", en MuÑoz (1998) p. 224.

30 Tornos (2000) p. 1330.
} 
doras independientes ${ }^{31}$. Estos órganos independientes que, como hemos dicho, nacen en el Derecho anglosajón con las Regulatory Agencies y constituyen la piedra angular de lo que Ariño denomina un "nuevo modelo de regulación para la competencia" 32.

Las autoridades reguladoras independientes, con un importante grado de experticia, asumen competencias para dictar normas concretas y especializadas, y tienen por objetivo último el buen funcionamiento del mercado, en armonía con la idea -que cada vez penetra con mayor fuerza- de la realización de los intereses públicos por intermedio de las fuerzas actuantes, en competencia, en el mercado ${ }^{33}$.

Desde esta perspectiva, De la Cruz expone que la motivación para una política de regulación tiene como punto de partida "las insuficiencias y fallos de mercados que observan los poderes públicos en el funcionamiento del sistema eléctrico" ${ }^{34}$, así como -complementa el Banco Mundial- "la preocupación por la equidad"35. Es decir, la regulación busca, por una parte, la corrección de las disfunciones del mercado y, por otra, la garantía de que el funcionamiento del sistema tienda al bienestar de la sociedad toda.

\section{4. ¿POR QUÉ REGULAR EL SECTOR ELÉCTRICO?}

En un sistema eléctrico que opera en libre concurrencia, la regulación debe preocuparse principalmente del libre funcionamiento de los mercados, la protección de los derechos del consumidor y la correcta actividad del sistema eléctrico. Asimismo, la regulación se justifica en cuanto constituir la energía un servicio de interés general que debe ser suministrado a toda la comunidad en determinadas condiciones de continuidad, seguridad y precios ${ }^{36}$.

De todas estas causas, centraremos el estudio en la regulación destinada al libre funcionamiento de los mercados. En este sentido, son los fallos de mercado que impiden

\footnotetext{
31 En Chile, el DL No 2224, de 1978, que creó la Comisión Nacional de Energía (CNE), organismo regulador de las actividades energéticas, como parte del proceso de liberalización del sector, con funciones equivalentes a las Regulatory Agencies anglosajonas. No obstante, como resulta evidente, sus facultades iniciales fueron limitadas, enmarcadas en un modelo de Estado chileno, centralizador de competencias y decisiones. Solo en los últimos años, ha habido un mayor contenido de las competencias de la CNE, que han aumentado su campo de acción en la toma de decisiones importantes en el modelo regulatorio eléctrico nacional. No obstante, no debemos olvidar que hace en marzo de 2013 el Panel de Expertos de la Ley General del Sector Eléctrico desautorizó las obras ordenadas por la CNE para conectar los Sistemas Interconectados Central y del Norte Grande, SIC y SING, respectivamente, como parte del Plan de Expansión del Sistema de Transmisión Troncal, en el dictamen 01-2013 que resolvió, entre otras, la discrepancia presentada por algunas empresas eléctricas y una Compañía Minera. Dicho dictamen se basó fundamentalmente en la falta de competencias de la CNE para ordenar dichas obras de conexión. Esta situación es un ejemplo de la contenida capacidad de actuación de la CNE en el modelo nacional, respecto del modelo de las Regulatory Agencies, en que no solo la regulación sino que toda la estrategia energética descansa precisamente en estos organismos especializados.

32 ARIÑo (2001) p. 560. Esta expresión tiene su raíz en la clásica idea de "regulation for competition" que nos recuerda CAMERON al expresar que "[...] As competition will not naturally occur in markets where natural monopolies of transportation exist, it is necessary for regulation to provide a surrogate for competition", CAMERON (2007) p. 30.

33 Parejo (2010-A) p. 62.

34 De la Cruz (2002) p. 147.

35 Banco Mundial (1997) p. 28.

36 Alfaro (2007) pp. 457 y ss.
} 
su libre actividad la primordial preocupación de la regulación energética. Los principales fallos de mercado dicen relación con: i) la situación de competencia imperfecta que se genera cuando existen rendimientos crecientes de escala o alcance como ocurre en la industria eléctrica (monopolios naturales); ii) las asimetrías informativas entre la industria y los consumidores (información imperfecta), y; iii) la existencia de externalidades ${ }^{37}$. Por ello, gran parte de la regulación del sector, así como las técnicas e instrumentos utilizados (como la separación de actividades o el acceso de terceros a las redes de transmisión) dice relación con la solución a estos fallos de mercado.

Asimismo, debe mencionarse que la regulación en el sentido que ha sido estudiado en los apartados anteriores, ha significado un explosivo desarrollo normativo, consolidando un ordenamiento energético abundante, especializado, y como característica propia del Derecho actual, esencialmente transitorio. Todo ello ha generado un conjunto de nuevos problemas, relacionados por ejemplo con la seguridad jurídica; esto es, cómo una regulación especializada y sectorial que busca resolver problemas de mercado concretos, y por tanto, con preceptos temporales, puede armonizarse con la necesaria seguridad jurídica que deben tener los actores del sistema eléctrico y los cambios de reglas del juego que se generan por el frecuente cambio normativo, considerando que la industria energética es intensiva en inversión.

Otro problema que ha desenmascarado la ciencia económica, tiene que ver con la captura del regulador. La excesiva relación y cercanía entre el ente regulador, organismo técnico y especializado, y las empresas reguladas, hace permeable al organismo público, hasta el punto en que el interés público que debe proteger el regulador cede ante el interés privado, y la regulación comienza a ser beneficiosa para la industria, obteniendo un trato más favorable, olvidando, en ocasiones, el interés público comprometido ${ }^{38}$.

Por último, debe considerarse lo que ha sido identificado por algunos autores como el paso de los fallos del mercado a los fallos del Estado, en referencia a las diversas debilidades que la excesiva regulación ha generado al sistema eléctrico, en aras de la corrección de los fallos del mercado, y que dicen relación, por ejemplo, con normas proteccionistas de los denominados campeones nacionales (grandes empresas eléctricas nacionales) ${ }^{39}$ o la pérdida de competitividad de los mercados por la imposición de objetivos de servicio públicos poco razonados o populistas ${ }^{40}$.

\section{NUEVO CONTENIDO DE LA REGULACIÓN}

Para identificar con mayor claridad el nuevo contenido de la regulación, se puede decir que en el modelo clásico de regulación para los grandes servicios públicos, la Admi-

\footnotetext{
37 Costas y Bel (1995) pp. 33 y ss.

38 La captura del regulador fue postulada por STIGLER (1971). Puede profundizarse sobre este tema en BoEHM (2005).

39 Un claro ejemplo de esta situación se dio en 2006, cuando los Gobierno de Rodríguez Zapatero y Merkel intervinieron abiertamente en la OPA presentada por E-ON para comprar ENDESA, sin resultados positivos, debido precisamente, a la oposición del gobierno español de que la empresa eléctrica española (uno de sus campeones nacionales, el otro es Iberdrola) quedara en manos de una empresa eléctrica alemana.

40 Soriano (2011) p. 87.
} 
nistración (el regulador) venía a sustituir al mercado y tomaba la mayoría de las decisiones: planificación, precios, decisiones de inversión, etc.; en cambio, la nueva regulación asume un rol de colaboración con los privados y su actuación principal tiene un doble objeto: la promoción de la competencia hasta donde sea posible y la protección de los intereses de los usuarios $^{41}$.

Pueden reconocerse, por tanto, como notas fundamentales de la función de regulación, en el sentido que tiene hoy ${ }^{42}$ :

a) Que no se trata solo de la actividad de dictar normas y controlar su correcta aplicación. La regulación incluye además de esta actividad, otras como la mediación o el arbitraje;

b) Que no se pretende dirigir la economía, sino buscar el equilibrio entre los diversos actores e intereses que intervienen en el mercado;

c) Que la actividad reguladora debe ser llevada a cabo por autoridades independientes y con conocimiento especializado en el sector que intervienen;

d) Que la regulación no es autorregulación. Exige una nota de alteridad de quien ejerce la función.

En lo que se refiere al tema energético, la regulación tiene especial interés ${ }^{43}$. El conjunto de características físicas, económicas y jurídicas -que hemos mencionado- de la electricidad y de la actividad que gira en torno a ella, permite afirmar que este sector estará siempre regulado (lo mismo ocurre con el transporte o las telecomunicaciones) ${ }^{44}$. Se trata de un mercado que necesita de la intervención del Estado, para que se garantice la correcta distribución de la energía como bien y como servicio, logrando un sistema eficiente económica y socialmente ${ }^{45}$. La regulación supone aquí la búsqueda del adecuado funcionamiento de un sistema complejo, el eléctrico ${ }^{46}$.

La libertad de entrada a la actividad energética, como elemento característico de la liberalización, que procura la producción eficiente de bienes y servicios en régimen de mercado, favoreciendo el suministro a los consumidores en condiciones adecuadas de calidad, precio y servicio, conduce a la multiplicación de normas reguladoras del marco jurídico

${ }^{41}$ ARIŃO (2001) p. 562.

42 Tornos (2010) pp. 101 y ss.

43 Cabe tener presente que el concepto regulación es un término ajeno al lenguaje jurídico. La primera aproximación de esta noción con el mundo del Derecho tuvo lugar con el sector audiovisual. Por tanto, los primeros y más importantes avances doctrinarios de la regulación desde un punto de vista jurídico tuvieron sus fuentes en el estudio de este sector. Jongen (1994); Casado (2008); Grupo de Regulación de las Telecomunicaciones (1998).

${ }^{4}$ Sobre esta afirmación, ARIÑo profundiza: "En efecto, la energía, el transporte, las telecomunicaciones o el sistema financiero estarán siempre regulados. La importancia social de tales actividades, la asimetría de posiciones entre empresas y usuarios, la dificultad de crear un mercado abierto y transparente, las limitaciones técnicas, y otros factores, así lo exigen", en Ariño (2001) p. 563.

${ }^{45}$ En este sentido, se puede observar que la propia LSE define las finalidades de la regulación para el sector. Expresa: "La regulación de dichas actividades tiene por finalidad: la adecuación del suministro de energía eléctrica a las necesidades de los consumidores, y la racionalización, eficiencia y optimización de aquellas, atendiendo a los principios de monopolio natural del transporte y la distribución, red única y de realización al menor coste".

46 Tornos (2010) p. 110. 
para garantizar, precisamente, la disminución de las barreras normativas, técnicas y económicas, para participar como agente en la actividad ${ }^{47}$.

En términos amplios, Hope y Singh han identificado como los objetivos propios de la regulación energética: i) la competencia; ii) la transparencia del mercado; iii) la protección del consumidor; iv) la eficiencia económica en el suministro; v) el suministro amistoso con el medio ambiente; vi) la seguridad de suministro, y; vii) la política de precios socialmente responsable. Ahora bien, en cuanto al cumplimiento de estos objetivos por los Estados miembros de la Unión Europea, puede observarse que los mayores cumplimientos están referidos a los objetivos propiamente de mercado (competencia, transparencia del mercado) y en menor medida a la protección del consumidor y la eficiencia económica del suministro, En cambio, el grado de incumplimiento aumenta en los objetivos ambientales, sociales y de seguridad de suministro, temas pendientes en la realización de una política energética común ${ }^{48}$.

Por su parte, González-Varas, haciendo referencia a los mercados de interés general -el eléctrico entre ellos-, resume de manera lúcida la necesidad de regulación de estos, así como el fundamental rol que esta forma de intervención estatal ha asumido en un sistema de mercado. Dice el autor: “En el fondo, la 'regulación' en estos casos es una manifestación de la especificidad de este tipo de mercados. No son estos simples mercados. De aquellos (los mercados de interés general) depende la realización de estos últimos (su abastecimiento, su suministro, su funcionamiento mismo); y están presentes ciertos intereses públicos de forma especialmente clara" ${ }^{49}$. En cuanto a lo que podría ser denominado como el "objetivo social" de la regulación, referido al logro de determinadas finalidades impuestas a los operadores de una actividad económica esencial como la eléctrica, por razones de interés general, Parejo aporta una interesante visión, cuando recuerda que la ampliación del interés legitimador de la regulación en el sector, más allá de la realización del mercado interior para la Unión Europea abierto y competitivo, encuentra su justificación en la promoción de la sostenibilidad del crecimiento en función de un alto nivel de calidad de vida, la protección del medio ambiente y la cohesión económica y social ${ }^{50}$.

Esto es, objetivos generales de carácter social, diversos del buen funcionamiento del sistema eléctrico o la liberalización de los mercados eléctricos, y la concreción del mercado interior para la electricidad en el caso de la Unión Europea, justifican que el Estado pueda regular la actividad, precisamente para el logro de dichos objetivos.

En este caso, el desarrollo normativo permitiría la coordinación de las diversas políticas públicas, y engarzar el modelo energético con el cumplimiento de objetivos superiores del Estado que tienen que ver con el bienestar de los ciudadanos, el cambio de paradigma de desarrollo desde un modelo propio de la era industrial hacia un modelo sostenible, o la garantía de acceso universal al suministro como expresión del principio de igualdad ${ }^{51}$.

47 Caballero (2003) p. 50.

48 Hope y Singh (2009) pp. 90 y ss.

49 González-Varas (2008) p.98.

50 Parejo (2010) p. 202.

51 Sobre el desarrollo actual del principio de igualdad véase: Díaz (2012) pp. 33-76. 
El conjunto de finalidades sociales constituye -desde nuestro punto de vista- un tema pendiente en la actividad reguladora de los organismos independientes, y especialmente del regulador energético. Este rol del Estado debe necesariamente ser armonizado con las garantías de los derechos de los ciudadanos exigibles al Estado en su concreción actual. Recordemos que en algún momento hemos identificado al Estado actual, que reemplaza al Estado prestador de servicios, como un Estado regulador y garantizador de los derechos de los ciudadanos. El contenido de los derechos amparados por la Constitución y las Leyes debe tener mayor importancia que su representación formal por los organismos reguladores independientes, y en este sentido, se debe avanzar.

\section{CONCURRENCIA DE COMPETENCIAS PARA LA REGULACIÓN DEL SECTOR ELÉCTRICO ESPAÑOL}

Identificados los principales problemas que buscan resolverse con la regulación: el correcto funcionamiento del sistema eléctrico y de su mercado ${ }^{52}$, la defensa de los derechos del consumidor y de la competencia, resulta necesario que la solución provenga también de diversos organismos de carácter público y privado, cuyo objeto consiste, precisamente, en la vigilancia y garantía de estos.

En este sentido, debe mencionarse que el ente regulador propio de los sistemas energéticos, la Comisión Nacional de Energía (CNE) posee competencias en todos los temas mencionados. No obstante, sus atribuciones han debido ser conciliadas con las de organismos reguladores especializados en temas propios de defensa de la libre competencia y los derechos del consumidor, que también poseen atribuciones en materia energética. Asimismo, España ha optado por entregar la operación del sistema y del mercado a organismos independientes, configurados como estructuras mercantiles propias del Derecho privado, pero que responden a intereses de servicio público y que están fuertemente reguladas por los organismos reguladores, a fin de garantizar, en última instancia, un sistema eléctrico competitivo, eficiente, seguro, y de precios accesibles.

A continuación revisaremos los principales objetivos de la regulación eléctrica en el modelo español.

\subsection{El CORRECTO FUNCIONAMIENTO DEL SISTEMA ELÉCTRICO}

Para garantizar el buen funcionamiento del sistema eléctrico, en el modelo español se ha entregado la gestión técnica y económica de este a organismos independientes, de carácter privado que asumen funciones de servicio público: el operador del sistema y el operador del mercado ${ }^{53}$.

\footnotetext{
52 La Ley 54/1997, expresa en el artículo 32: "Para asegurar el correcto funcionamiento del sistema eléctrico dentro del marco que establece la presente Ley, corresponde al operador del mercado y operador del sistema, respectivamente, asumir las funciones necesarias para realizar la gestión económica referida al eficaz desarrollo del mercado de producción de electricidad y la garantía de la gestión técnica del sistema eléctrico".

53 En este sentido, De la QuAdra-SAlCEDo señala que: "El operador del mercado y del sistema ejercen en realidad funciones públicas. Funciones reservadas a unas entidades que ninguna otra puede ejercer. No cabe, pues, iniciativa privada para ejercer tales actividades. Las mercantiles a las que la Ley atribuye esas funciones
} 
En cuanto a la operación técnica del sistema eléctrico, es necesario distinguir entre la gestión de la red de transporte y la gestión de las redes de distribución. La primera es entregada a Red Eléctrica de España S.A. (REE), propietaria de todas las redes de transporte en el Estado español. Este modelo permite avanzar en dos técnicas clave de introducción de competencia en actividades naturalmente monopólicas como el transporte de electricidad:

i) La separación de actividades. En este sentido, la fase de transporte de electricidad se desvincula totalmente de las otras fases de la actividad eléctrica, siendo entregada la propiedad de las redes de transporte a REE, permitiendo que la gestión de la red se realice con independencia de los intereses de las empresas generadoras, distribuidoras y comercializadoras; asimismo el desarrollo y ampliación de la red de alta tensión se realiza con criterios objetivos y de interés general, permitiendo un mejor funcionamiento del sistema eléctrico;

ii) El acceso de terceros a la red. Esta técnica para introducir competencia en el sistema eléctrico, denominada Third Party Access, permite el acceso de terceros a la red operada por una empresa concreta, a fin de que pueda transportarse electricidad desde un punto de generación a otro de consumo, por redes de propiedad de otro, previo pago de una tarifa, promoviendo la competencia. Esta técnica ha sido desplegada por el modelo liberalizador que la Unión Europea ha propuesto para los Estados miembros de la Unión Europea, y España la ha integrado a su legislación a través de sucesivas reformas a la Ley 54/1997, del Sector Eléctrico ${ }^{54}$. En España, REE, como empresa propietaria de las redes de transporte y operadora del sistema, asume el deber de permitir el acceso a las redes de todos aquellos operadores que deseen transportar electricidad, si las condiciones técnicas lo permiten, promoviendo -en consecuencia- la competencia en el sistema ${ }^{55}$.

Por el contrario, la gestión de la distribución de la electricidad es entregada a los propios distribuidores, que asumen funciones públicas referidas a la explotación, el mantenimiento y el desarrollo de sus propias redes de distribución, con garantía del principio de acceso de terceros a las redes, de conformidad con lo indicado en el artículo 39.1 de la Ley 54/1997 del Sector Eléctrico.

El objetivo final de este modelo de operación del sistema asumido por la regulación española del sector es que cada consumidor final pueda contratar la electricidad con cualquier proveedor conectado al mercado interior, logrando mejores precios sin afectar la calidad del servicio.

\subsection{EL BUEN FUNCIONAMIENTO DEL MERCADO ELÉCTRICO}

La operación del mercado eléctrico ibérico es entregada al Operador Ibérico de Energía, Polo Español, S.A. (OMIE) $)^{56}$, quien asume la gestión conjunta de los mercados español y portugués. El mercado es estructurado en mercado diario, mercado intradiario, mercado a plazo y mercado de servicios de ajuste. Según expresa la Resolución de 23 de

públicas no se integran en el aparato del Estado como un órgano mismo, pero, desde luego, no son una sociedad anónima cualquiera”, De LA Quadra-SAlcedo (1998) pp. 603 y ss.

54 Trillo-Figueroa Martínez-Conde (1998) pp. 37-83.

55 Para profundizar en este tema, véase De La CruZ y Ónega (2006) pp. 606-620.

56 Desde el 1 de julio de 2011 el operador del mercado eléctrico español se fusionó con el operador mercado eléctrico portugués, dando paso al mercado ibérico, gestionado por un operador de común. 
julio de 2012, de la Secretaría de Estado de Energía, por la que se aprueban las reglas de funcionamiento del mercado diario e intradiario de producción de energía eléctrica:

"En el mercado se llevan a cabo las transacciones de compra y venta de energía eléctrica para el día siguiente. [...] También puede producirse en el Mercado diario la entrega física de la energía negociada en los Mercados organizados a plazo.

El Mercado intradiario, tiene por objeto atender la oferta y la demanda de energía que se pueden producir, en las horas siguientes, con posterioridad a haberse fijado el Programa Diario Viable.

El Mercado de servicios de ajuste incluye a todos aquellos servicios que, teniendo carácter potestativo, los Operadores del Sistema consideren necesarios para asegurar el funcionamiento del sistema $[\ldots]$ ". (Regla $\left.1^{\mathrm{a}}\right)$.

Finalmente, en el mercado a plazo, se realizan transacciones de compra y venta de energía eléctrica para periodos superiores a los pactados en los mercados antes descritos.

En general, el funcionamiento del mercado, en los términos indicados, requiere de una profusa regulación a cargo de la Comisión Nacional de Energía, que es aplicada por el Operador del Mercado, a fin de permitir el correcto desarrollo económico de los mercados mayorista y minorista de electricidad, en un modelo de libre concurrencia.

\subsection{LA DEFENSA DEL CONSUMIDOR}

En cuanto a la defensa de los derechos del consumidor, debemos indicar que el modelo actual, no ha permitido el avance de organismos autónomos de defensa de estos intereses en materia energética, optándose por reforzar las funciones de los entes reguladores energéticos. En este sentido, la Carta Europea de los Derechos de los Consumidores de Energía, de $2008^{57}$, subraya que las autoridades reguladoras (energéticas) nacionales deben contar con suficientes competencias estatutarias y recursos para la protección de los consumidores, incluido el derecho a imponer sanciones a los proveedores que incumplan normas referentes a esta cuestión (puntos 33 y siguientes).

Hay mucho por hacer en este tema, y sin duda, constituye una cuestión sobre la que se debe trabajar en profundidad para que puedan lograrse avances en la materia. La dificultad en la comprensión de las características técnicas y económicas del sistema eléctrico impide el empoderamiento de las organizaciones ciudadanas de defensa de los derechos del consumidor en la protección de sus derechos en materia energética ${ }^{58}$.

\footnotetext{
57 Resolución del Parlamento Europeo, de 19 de junio de 2008, sobre una Carta Europea de los Derechos de los Consumidores (2008/2006(INI)).

58 En nuestro país la situación es muy similar, por cuanto el Servicio Nacional del Consumidor (SERNAC) y las Asociaciones de Consumidores tampoco poseen la experticia para defender los derechos de los consumidores en un tema tan técnico y sectorial como el energético, y el modelo chileno ampara los derechos de estos a través de facultades conferidas, principalmente, a la Superintendencia de Electricidad y Combustibles (SEC). Véase la Ley 18410 de 1985, art. 3º. También, la Comisión Nacional de Energía, que originariamente no se contemplaba con un organismo defensor de los derechos de los consumidores, ha avanzado muy sutilmente a través de la Resolución exenta No 440, de 8 de agosto de 2011, que, en este sentido: i) establece un Consejo de la Sociedad Civil, integrado por diversas asociaciones sin fines de lucro, entre ellos un representante de los
} 


\subsection{LA PROTECCIÓN DE LA LIBRE COMPETENCIA}

Contrario a lo que sucede en la defensa del consumidor, la regulación (considerada aquí como la actividad propia del organismo regulador de los sistemas energéticos) y la protección de la competencia sí han logrado puntos de encuentro. "La regulación y defensa de la competencia son herramientas complementarias que permiten encontrar el balance entre la protección de los consumidores y los incentivos de las empresas. Asimismo, "a medida que el mercado ha ido consolidándose y la regulación ha ido ajustándose a las nuevas necesidades, la defensa de la competencia ha ido adquiriendo cada vez un papel más relevante" ${ }^{\text {59 }}$.

De Hauteclocque señala que, del examen del sector energético, pueden identificarse importantes objetivos para la legislación de defensa de la competencia (antitrust law): concentración de mercado, barreras de entrada, integración de los mercados, problemas de transparencia e información, formación de precios, y contratos a largo plazo que producen captura del consumidor ${ }^{60}$. Estos fallos de mercado, son abordados tanto por el regulador sectorial (Comisión Nacional de la Competencia, CNE), como por la autoridad de defensa de la competencia.

Se explica esta concurrencia de competencias en la multiplicidad de atribuciones asignadas a los órganos reguladores energéticos: no solo deben velar por el funcionamiento del sistema eléctrico, sino también por la defensa de los consumidores y la promoción de la competencia. Es en este último objetivo, donde las facultades del ente regulador coinciden con las atribuciones conferidas al sistema de defensa de la competencia que desarrollan los Estados. Aun cuando parece más o menos claro a nivel europeo, que se trata de competencias complementarias para cumplir un fin prioritario, el establecimiento de un mercado interior para la Unión Europea.

En este sentido, apunta Cameron: "The principal role of the competition law in energy market reform has been to provide support to the development of a sector-specific regime" ${ }^{\text {" }}$. Desde el principio, la función principal asignada por la Comisión Europea a las autoridades nacionales de regulación energética ha sido la de llevar a cabo la liberalización de los sectores energéticos, asumiendo las autoridades de la competencia labores de vigilancia de su adecuada aplicación.

Varios modelos han buscado articular la regulación sectorial y la defensa de la competencia en el estratégico sector energético; mencionaremos dos. En primer lugar, Estados Unidos ha definido desde hace ya bastante tiempo que las conductas plasmadas en la regulación sectorial no pueden ser perseguidas desde la perspectiva del Derecho antimonopolios (Antitrust). Un segundo modelo es el utilizado por la Unión Europea, en el que prima el principio de especialidad. Es decir, lex specialis derogat generalis. Si un operador realiza una

consumidores de los mercados energéticos (art. 6), como órgano consultivo de la CNE, y; ii) Establece procesos de consulta ciudadana (no obligatorios) respecto de políticas, planes, programas y/o proyectos que desarrolle o pueda desarrollar en el ámbito de sus competencias (art. 4\%).

59 Agosti y Pardilla (2009) pp. 149 y ss.

60 Hauteclocque (2008) pp. 92 y ss.

61 Cameron (2007) p. 564. 
conducta autorizada expresamente por una normativa sectorial, dicha conducta conforme a derecho, no podrá ser perseguido en el ámbito del Derecho de la Competencia ${ }^{62}$.

Este último método es el que ha utilizado el ordenamiento español para resolver eventuales conflictos entre el organismo regulador de la energía y la autoridad de defensa de la competencia, elaborando dos criterios: i) el primero de ellos, dice relación con que la CNE habitualmente tiene una intervención ex ante y los órganos de defensa de la competencia, por regla general, tienen una actuación ex post, ii) la asunción de una competencia horizontal y centralizada por parte de Comisión Nacional de la Competencia $(\mathrm{CNC})^{63}$.

Asimismo, hay algunas normas concretas que intentan coordinar las competencias de la CNC y la CNE. Así por ejemplo, cuando se trata de conductas prohibidas en la Ley de Defensa de la Competencia ${ }^{64}$, la Ley 34/1998, del sector de hidrocarburos, en la disposición adicional undécima, expresa que son funciones de la CNE: "velar para que los sujetos que actúan en los mercados energéticos lleven a cabo su actividad respetando los principios de libre competencia. A estos efectos, cuando la Comisión detecte la existencia de indicios de prácticas restrictivas de la competencia prohibidas por la Ley 16/1989, de 17 de julio, de Defensa de la Competencia, lo pondrá en conocimiento del Servicio de Defensa de la Competencia, aportando todos los elementos de hecho a su alcance y, en su caso, un dictamen no vinculante de la calificación que le merecen dichos hechos" (función duodécima).

Esta exigencia de comunicación permite una clara distinción de atribuciones para cada organismo, dentro de una competencia compartida, referida al respeto de los principios de la libre competencia. No obstante, advierte Bacigalupo que la CNE ha expresado en ocasiones la necesidad de ampliar sus atribuciones a la instrucción de expedientes en materia de defensa de la competencia, dado el conocimiento que tiene del sector. El argumento dado para no aceptar esta sugerencia, es que, precisamente, la cercanía al sector podría darle una visión distorsionada ${ }^{65}$.

No obstante, "con independencia del modelo de reparto de funciones por el que se opte, resulta indiscutible en todo caso que la atribución a los reguladores de una función de supervisión de los sectores es perfectamente adecuada a su naturaleza y no comporta contradicción alguna con el esquema de ejercicio de potestades de manera exclusiva por parte de las autoridades de competencia. Por el contrario, constituye un complemento no solo adecuado sino necesario, dado que la mera aplicación a posteriori de las normas de competencia por las autoridades encargadas es insuficiente, debiendo completarse por el regulador tanto con una labor regulatoria preventiva como con una labor de supervisión de conductas que aquellas autoridades, por sí mismas o debido a las denuncias de particulares, pudieran no haber detectado" 66 .

Por otra parte, una peculiaridad del sistema eléctrico, es que coexisten actividades libres y actividades reguladas, es decir, mientras que en el modelo energético de la Unión Eu-

\footnotetext{
62 Sala (2010) p. 130.

${ }^{63}$ Alfaro (2007) pp. 464 y ss.; Sala (2010) pp. 130-131.

64 Real Decreto Legislativo 1/2007.

65 Bacigalupo (2009-B) pp. 136-137.

66 Salinas (2007) p. 202.
} 
ropea las fases de generación y comercialización se desarrollan bajo los principios de libertad de empresa; la operación del sistema, el transporte y la distribución tienen el carácter de actividades reguladas. Este elemento estructural del mercado eléctrico ha generado algunas complicaciones al momento en que las autoridades de la competencia puedan controlar y supervisar que en este mercado se cumplan las normas de defensa de la competencia, por cuanto la tendencia a la concentración de estas actividades que deben funcionar de manera separada, dificulta la identificación de las conductas que pueden o no ser sancionadas por la $\mathrm{CNC}^{67}$.

Finalmente, diremos que en España, las competencias de defensa de la competencia de la CNE son esencialmente preventivas; no posee facultades para sancionar conductas antimonopólicas, por lo que hasta ahora no han existido grandes conflictos de competencias con las autoridades de defensa de la competencia.

\section{CONCLUSIONES}

1) La regulación en su concepción moderna, proveniente del mundo anglosajón, se ha transformado en el instrumento de intervención propio del nuevo paradigma económico, que promueve la liberalización de los servicios económicos esenciales y el funcionamiento del mercado como medio de asignación de los recursos. Respecto de la industria energética, históricamente reticente al funcionamiento del mercado, la regulación ha permitido la transformación de las estructuras del sector eléctrico, para introducir competencia y promover el desarrollo de un mercado energético interior en la Unión Europea (UE). Puede plantearse en este sentido, que la privatización de la industria eléctrica emprendida en países como Chile, Perú o Colombia, puede ir acompañado de una estrategia de liberalización de los mercados nacionales de los Estados latinoamericanos, para la implantación de un mercado eléctrico sudamericano que permita a los consumidores de cada país, poder elegir entre diversos suministradores de este gran mercado, que provocaría una mejor calidad del servicio, mayor seguridad energética, y mejores precios, aunque esto último no ha sido realmente efectivo en el mercado interior de la UE, que teóricamente funciona desde 2007.

2) Se pueden identificar cuatro objetivos generales que la regulación propuesta por la Unión europea ha expresado para el sector eléctrico, y que España ha integrado a su modelo energético: el funcionamiento del sistema eléctrico, el funcionamiento del mercado eléctrico, la protección del consumidor y la defensa de la libre competencia. En este contexto, el Estado asume un rol de garante de los derechos de las personas y regulador de este sistema económico, labor que desarrolla a través de un organismo autónomo y técnico, la Comisión Nacional de Energía.

El primero de los objetivos, conlleva las ideas de suministro eléctrico continuo, seguro, a precios bajos y amigables con el medio ambiente. En este sentido, el modelo implementado por España, según el diseño promovido por la Unión Europea, contempla la existencia de un operador de sistema (Red Eléctrica de España S.A.) que gestiona las redes de transporte, con el objetivo final de promover un modelo liberalizado para el sector eléctrico.

67 Guillén (2007) pp. 247 y ss. 
Por otra parte, el funcionamiento del mercado, es entregado a un operador de mercado (Operador Ibérico de Energía, Polo Español S.A.), que en última instancia garantiza que exista en todo momento la provisión de electricidad que los consumidores demanden. El correcto funcionamiento del mercado eléctrico, es la función que requiere con mayor intensidad de la actividad del organismo regulador (CNE) y del organismo de defensa de la competencia (CNC). Las estructuras de empresas integradas verticalmente en torno a una actividad de transporte (industria de redes) organizada como monopolio natural han necesitado de una fuerte regulación y ha presentado serias dificultades a la hora de implantar el mercado. No obstante, a través de instrumentos y ficciones jurídicas como el acceso de terceros a las redes y la separación de actividades se ha logrado introducir los principios de libertad y de competencia en este complejo sector y reducir los fallos de mercado.

En cambio, la protección del consumidor sigue siendo una tarea pendiente en el modelo energético que se impone desde la liberalización del sector. Debe haber un mayor esfuerzo de la Administración en orden no solo a regular para lograr una mayor defensa de los derechos del consumidor, sino que deben crearse instrumentos eficaces para el logro de este objetivo. La asimetría de la información que posee el consumidor respecto de los suministradores dificulta enormemente el empoderamiento del consumidor como actor clave del sistema eléctrico.

Finalmente, la defensa de la competencia ha requerido de una labor de delimitación de las competencias de la Comisión Nacional de Energía, como organismo regulador especializado con atribuciones en materia de libre competencia, y la Comisión Nacional de la Competencia, como organismo regulador en materia de defensa de la libre competencia. En este sentido, se ha decidido por concentrar las competencias preventivas en la CNE y las competencias represivas, en la $\mathrm{CNC}$, aun cuando, esta última mantiene atribuciones de carácter preventivo.

3) La asunción de organismos reguladores independientes en el modelo energético ha permitido separar la actividad reguladora del Estado de la prestación del servicio. Se trata de un gran avance en el desarrollo de los mercados eléctricos, al punto que rápidamente los organismos reguladores han pasado a ser garantes del nuevo modelo. No obstante, sigue siendo un problema pendiente de resolución la captura del regulador, que permea su independencia y beneficia a la industria eléctrica con organismos proclives a desarrollar regulaciones pactadas con la industria.

4) La concurrencia de competencias del ente regulador con el organismo de defensa de la competencia no puede restringir el campo de acción de ambos, sino que debe fomentar la cooperación a fin de lograr el correcto funcionamiento del mercado, que sustenta el modelo energético actual.

5) Finalmente, este trabajo, abre la puerta al desarrollo de nuevas investigaciones orientadas a la regulación del sector eléctrico chileno en un entorno de libre competencia, y el desarrollo de un mercado regional de electricidad en Sudamérica (que ya se esbozó en la conclusión 1a) que permita a los consumidores ejercer la posibilidad de elección del suministrador. 


\section{BIBLIOGRAFÍA CITADA}

Agosti, Luis y Padilla, Jorge (2009): "Precios excesivos", en: Guillén Caramés, Javier (dir.), Derecho de la Competencia y energía eléctrica (Cizur Menor, Thomson Reuters) pp. 145-193.

Alfaro Águila-Real, Jesús (2009): "Las autoridades de competencia nacionales ante los sectores regulados: la aplicación de la ley de defensa de la competencia al sector de la energía. La coordinación entre los organismos reguladores", en: BECKER, Fernando et al. (dir.), Tratado de la Regulación del Sector Eléctrico. Aspectos Jurídicos (Tomo I) (Cizur Menor, Aranzadi) pp. 457-479.

Aranzadi Martínez, Claudio (2009): "La singularidad económica del sector eléctrico", en: BeCKer, Fernando et al. (dir.), Tratado la Regulación del Sector Eléctrico. Aspectos Económicos (Tomo II) (Cizur Menor, Aranzadi) pp. 99-120.

Ariño Ortiz, Gaspar (2010): "Problemas de competencia en los sectores energéticos", en: Serrano González, M. y Bacigalupo Saggese, M. (dir.), Cuestiones actuales del Derecho de la Energía. Regulación, Competencia y Control Judicial (Madrid, CNE-Iustel) pp. 135-162.

Ariño Ortiz, Gaspar (2006): Energía en España y desafio europeo. Seguridad, concentración empresarial, competencia, regulación (Granada: Comares) 417 pp.

Ariño Ortiz, Gaspar (2001): Principios de Derecho Público Económico. Modelo de Estado, Gestión Pública, Regulación Económica (2a ed.) (Granada, Comares) 1089 pp.

Ariño Ortiz, Gaspar y López de Castro, Lucía (1998): El sistema eléctrico español: Regulación y competencia (Madrid, Montecorvo) 680 pp.

Ariño Ortiz, Gaspar (1993): Economía y Estado. Crisis y reforma del sector público (Madrid, Marcial Pons) 430 pp.

Bacigalupo Saggese, Mariano (2009-A): "Electricidad, servicio público y mercado", en Becker, Fernando et al. (dir.), Tratado de la Regulación del Sector Eléctrico. Aspectos Jurídicos (Tomo I), (Cizur Menor, Aranzadi) pp. 87-110.

Bacigalupo Saggese, Mariano (2009-B): "La coordinación y delimitación de funciones entre las autoridades de defensa de la competencia y el regulador sectorial energético", en: Muñoz Machado, Santiago et al., Derecho de la regulación económica. Sector energético (Tomo I), (Madrid, Iustel) pp. 125-153.

Banco Mundial (1997): El Estado en un mundo de transformaciones (Washington D.C., Banco Mundial).

Boenм, Frédéric (2005): "Corrupción y captura en la regulación de los servicios públicos", Revista de Economía Institucional, vol. VII, No 13: pp. 245-263.

Caballero SÁnchez, Rafael (2003): Infraestructuras en red y liberalización de servicios públicos (Madrid, Instituto Nacional de Administración Pública) 454 pp.

Cameron, Peter (2007): Competition in Energy Markets. Law and regulation in the European Union (2a ed.) (Oxford, Oxford University Press) 478 pp.

Casado Casado, Lucía (2008): "La configuración de la televisión de titularidad del estado como servicio público en la ley 17/2006, de 5 de junio, de la radio y la televisión de titularidad estatal", Revista Vasca de Administración Pública, No 80: pp. 55-109. 
Cassagne, Juan (1996): "El resurgimiento del servicio público y su adaptación en los sistemas de economía de mercado (Hacia una nueva concepción)", Revista de Administración Pública, No 140: pp. 95-110.

CHIck, Martin (2007): Electricity and Energy policy in Britain, France and the United States since 1945 (Cheltenham, Edward Elgar Publishing) 193 pp.

Costas, Antón y BeL, Germà (1995): "Regulación y desregulación en la economía europea actual”, en García Delgado, José; Pedreño Muñoz, Andrés y Velarde Fuertes, Juan (coord.), Regulación y competencia de la economía española: IX Jornadas de Alicante sobre economía española (Madrid, Civitas) pp. 33-64.

De Hauteclocque, A. (2008): "EC Antitrust Enforcement in the Aftermath of the Energy Sector Inquiry: a Focus on Long-Term Supply Contracts in Electricity and Gas", en, Delvaux, Bram; Hunt, Michael y Talus, Kim (Ed.), EU Energy Law and Policy Issues (Rixensart, Euroconfidentiel) pp. 91-108.

De la Cruz Ferrer, Juan (2002): Principios de regulación económica en la Unión Europea (Madrid, Instituto de Estudios Económicos) 358 pp.

De la Cruz Ferrer, Juan (1999): La liberalización de los servicios públicos y el sector eléctrico: Modelos y análisis de la Ley 54/1997 (Madrid, Marcial Pons) 503 pp.

De la Cruz Ferrer, Juan y Ónega Carrión, Nuria (2006): "Comentarios al artículo 38 de la Ley del Sector Eléctrico”, en López-Ibor Mayor, Juan y Beneyto Pérez, José, Comentarios a las leyes eléctricas. Ley del Sector Eléctrico (Tomo I), (Cizur Menor, Aranzadi) pp. 606-620.

Díaz García, Iván (2012): "Igualdad en la aplicación de la ley. Concepto, iusfundamentalidad y consecuencias", Revista Ius et Praxis, vol. XVIII, No 2: pp. 33-76.

Fabra Utray, Jorge (2004): ¿Liberalización o regulación? Un mercado para la electricidad (Madrid, Marcial Pons) 438 pp.

Fúnez Guerra, C.; Almansa Rodríguez, E. y Fuentes Ferrera, D. (2010): "El hidrógeno: vector energético del futuro", Energía \& Minas, No 8: pp. 20-27.

Gimeno Feliú, José (1994): El sector eléctrico como servicio público (Madrid, Civitas) pp. 451.

GonZÁlez-Varas IbáÑEZ, Santiago (2008): Tratado de Derecho Administrativo: Derecho Administrativo Económico (Tomo VI) (Cizur Menor, Aranzadi) 3000 pp.

Grupo de Regulación de las Telecomunicaciones (GRETEL) (1998): Competencia y regulación en los mercados de las telecomunicaciones, el audiovisual e Internet (Madrid, Colegio Oficial de Ingenieros de Telecomunicaciones) $335 \mathrm{pp}$.

Guillén Caramés, Javier (2009): "El abuso de posición de dominio en el sector eléctrico", en Guillén Caramés, Javier (Director), Derecho de la Competencia y energía eléctrica, (Cizur Menor, Thomson Reuters) 398 pp.

Hope, E. y Singh, B. (2009): "Harmonizing an effective regulation in Europe", en GlaCHANT, Jean-Michel y LÉVÊQUe, François, Electricity reform in Europe, (Cheltenham, Edward Elgar Publishing) pp. 89-104.

Jongen, F. (1994): La police de l'audiovisuel. Analyse comparée de la régulation de la radio et de la télévision en Europe (Bruxelles, Bruylant, Bruxelles) 544 p. 
Medlock, Kenneth (2009): "The economics of energy supply", en Evans, Joanne y Hunt, Lester (Editors), International Handbook on the Economics of Energy, (Edward Cheltenham, Elgar Publishing) pp. 51-72.

Mir Puigrelat, Oriol (2004): Globalización, Estado y Derecho. Las transformaciones recientes del Derecho Administrativo (Civitas, Madrid) 283 pp.

Muñoz Machado, Santiago (1998): Servicio público y Mercado. Los fundamentos (vol. I) (Madrid, Civitas) 1050 pp.

Muñoz Machado, Santiago (2010): Tratado de Derecho Administrativo y Derecho Público General. La actividad administrativa (Tomo IV) (Madrid, Iustel), 1128 pp.

Nebreda Pérez, Joaquín (1999): Distribución eléctrica. Concurrencia de disciplinas jurídicas (Madrid, Civitas) 1028 pp.

Parejo Alfonso, Luciano (2009): "La intervención administrativa de las empresas energéticas", en Muñoz Machado, S. et al., Derecho de la regulación económica. Sector energético (Tomo I), (Madrid, Iustel) p. 201-231.

Parejo Alfonso, Luciano (2010-A): "Las relaciones y delimitación entre regulador independiente y la Administración General del Estado", en: Serrano GonzÁlez, M. y Bacigalupo Saggese, M. (dir.), Cuestiones actuales del Derecho de la Energía. Regulación, Competencia y Control Judicial (Madrid, Iustel) 59-106.

Parejo Alfonso, Luciano et al. (2010-B): Lecciones de Derecho Administrativo. Orden económico y sectores de referencia (Valencia, Tirant lo Blanch) 790 pp.

Real Academia Española (2011): Diccionario de la lengua española (22a ed.) (Madrid, Espasa Calpe) $1672 \mathrm{pp}$.

Rivero Torre, Pedro (2009): "El suministro de electricidad", en: Becker, Fernando et al. (dir.), Tratado de la Regulación del Sector Eléctrico. Aspectos Económicos (Tomo II), (Cizur Menor, Aranzadi) pp. 79-98.

Sala Arquer, J. (2010) "Las relaciones y delimitación de funciones entre el regulador de la energía independiente y las autoridades de defensa de la competencia”, en: SERRANO González, M. y Bacigalupo Saggese, M. (dir.), Cuestiones actuales del Derecho de la Energía. Regulación, Competencia y Control Judicial (Madrid, Iustel) pp. 127-134.

Salas Hernández, J. (1977) Régimen jurídico-administrativo de la energía eléctrica (Bolonia, Publicaciones del Real Colegio de España) 502 pp.

Salinas la Casta, E. (2007): "Comentario de las nuevas funciones de supervisión de la CNE y de la nueva redacción de la función decimocuarta”, en: LÓPEZ-IBOR MAYOR, Vicente (coord.), Comentarios a la Ley de reforma al sector eléctrico (Ley 17 de 2007) (Cizur Menor, Aranzadi) pp. 189-254.

Soriano García, José (2011): "Liberalización económica, Sector Público y Derecho Administrativo", Revista parlamentaria de la Asamblea de Madrid, No 24: pp. 79-234.

Stigler, G. J. (1971): “The Theory of Economic Regulation”, Bell Journal of Economics and Management Science, Vol. 2, No 1: pp. 3-20.

Thomé, Henrique (2001): "Privatización: Algunas cuestiones en debate", Hitos de Ciencias Económico Administrativas, Vol. 7, No 18: pp. 33-40.

Tornos Más, Joaquín (2010): "El regulador de la energía y otros reguladores sectoriales: Comparación de modelos”, en Serrano González y Bacigalupo Saggese (directores), 
Cuestiones actuales del Derecho de la Energía. Regulación, Competencia y Control Judicial (Madrid, CNE-Iustel) pp. 107-127.

Tornos MAs, Joaquín (2000): "La actividad de regulación", en: Sosa WaGner, F. (Coordinador), El Derecho Administrativo en el umbral del siglo XXI. Homenaje al Profesor Dr. D. Ramón Martín Mateo (Tomo III) (Tirant lo Blanch, Valencia) 1329-1342.

Trillo-Figueroa Martínez-Conde, Jesús (1998): "Liberalización eléctrica en España: Aspectos básicos de su regulación”, en Neвot Lozano, José et al. Competencia y sector eléctrico: un nuevo régimen Jurídico (Madrid, Civitas) pp. 37-83.

Trillo-Figueroa Martínez-Conde, Jesús y López-Jurado Escribano, Francisco (1996): La regulación del sector eléctrico (Madrid, Civitas) $584 \mathrm{pp}$.

Vergara Blanco, Alejandro (2004): Derecho Eléctrico (Santiago, Ed. Jurídica) 439 pp.

\section{NORMAS CITADAS}

DFL No 1, del Ministerio de Minería, de 1982. Aprueba modificaciones al DFL No 4 de 1959, Ley General de Servicios Eléctricos, en Materia de Energía Eléctrica.

Directiva 2009/72/CE, de 13 de julio de 2009, sobre normas comunes para el mercado interior de la electricidad y por la que deroga la Directiva 2003/54/CE.

Directiva 2003/54/CE, de 26 de junio de 2003, sobre normas comunes para el mercado interior de la electricidad y por la que deroga la Directiva 96/92/CE.

Directiva 96/92/CE, de 19 de diciembre de 1996, sobre normas comunes para el mercado interior de la electricidad.

DL No 2224, de 8 de junio de 1978, que crea la Comisión Nacional de Energía.

Ley 54/1997, de 27 de noviembre, del sector eléctrico.

Ley de 22-VI-1898, que crea el impuesto al consumo de fluido eléctrico.

Ley de 23-III-1900, de servidumbre forzosa de paso de corrientes eléctricas, desarrollada por el RD de 15-VI-1901.

Ley de expropiación forzosa, de 23-III- 1900.

DL No 2224, de 8 de junio de 1978 creó la Comisión Nacional de Energía.

RD 28-V-1896, sobre contribución industrial.

RD de 25-I- 1901, que regula los contadores eléctricos.

RD de 22-XI-1912 que crea la Comisión Permanente Española de Electricidad.

Real Decreto Legislativo 1/2007, de 16 de noviembre, por el que se aprueba el texto refundido de la Ley General para la Defensa de los Consumidores y Usuarios y otras leyes complementarias. 